\title{
The emperor of all maladies: a biography of cancer
}

\section{Dong Hoon Suh}

Department of Obstetrics \& Gynecology, Seoul National University College of Medicine, Seoul, Korea sdhwcj@naver.com

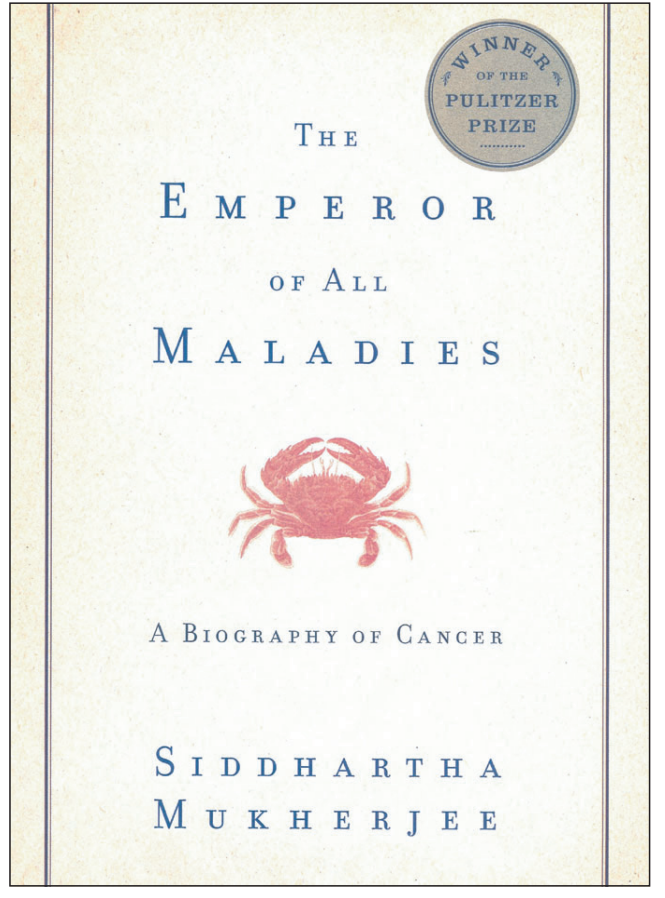

By Siddhartha Mukherjee

New York: Scribner; 2010

ISBN: 978-1-4391-0795-9

Hardcover, 571 pages
I found this book quite unique in that a history of cancer was covered along with a detailed description of personal experience during the author's early days as an oncology fellow. As the same position of an oncology fellow and researcher, I read the book in one sitting because I could empathize with the author all the way through reading the book. The ultimate purpose of this book is not just to understand the past of cancer but to raise a question: is it possible to eradicate this disease from our bodies and societies forever?

To try to help the readers come up with the answer, this 571page book covers the whole story of fighting against cancer from its first documented appearances to the recent remarkable triumphs including Trastuzumab for Her-2 expressing breast cancer and Imatinib for Bcr-abl positive chronic myelogenous leukemia. Notably, historical photos were illustrated so that the readers can see at a glance the story of this book.

This book is composed of six parts that deal with the cenotaphic events according to the historical progress in the screening, diagnosis, treatment, and prevention of cancer. In the first part, the author mentioned the first description of leukemia as "a suppuration of blood" by Dr. John Bennett and subsequent renaming to "leukemia" by Dr. Rudolf Virchow in 1847. The author introduces Dr. Sidney Farber as the father of modern chemotherapy, who accidentally discovered aminopterin as a powerful anti-cancer chemical leading to the dramatic remission of leukemia. In addition, he describes the development of surgery and anesthesia as well as the concept of radicality in the removal of the tumor. In the following parts, this book covers several landmarks in the development of the treatment of cancer including combination chemotherapy and molecular targeted agents. Another part depicts the exploration history of cancer causes which includes the discovery of cancer-causing viruses such as Rous sarcoma virus and two-hit hypothesis, that is, activated proto-oncogenes 
(jammed accelerators) and inactivated tumor suppressor genes (missing brakes).

From a clinical viewpoint of a gynecologic oncology fellow, I have particularly welcomed part four, "prevention is the cure". The author provides the history of Papanicolau test and mammography as the effective screening strategies for uterine cervix and breast cancers, respectively. To stop smoking is also introduced as an effective primary prevention of lung cancer.

In conclusion, this book sheds new light on the future of "war against cancer" through comprehensively reviewing the past history of it. I believe this book will provide hope and clarity to those seeking to demystify cancer. I strongly recommend this book not only to young oncology researchers but also to the health policymakers who are in charge of government money plan.

"We are so close to a cure for cancer. We lack only the will and the kind of money and comprehensive planning that went into putting a man on the moon" $\quad$-Dr. Sidney Farber- 